\title{
Controller Application of a Multi-Layer Quantum Neural Network with Qubit Neurons*
}

\author{
Kazuhiko TAKAHASHI ${ }^{* *}$, Motoki KUROKAWA*** and Masafumi HASHIMOTO ${ }^{\dagger}$ \\ ** Department of Information Systems Design, Doshisha University \\ 1-3 Miyakodani Tatara, Kyotanabe, Kyoto 610-0321, Japan \\ E-mail: katakaha@mail.doshisha.ac.jp \\ *** Graduate school of Doshisha University (currently Canon Inc.) \\ 1-3 Miyakodani Tatara, Kyotanabe, Kyoto 610-0321, Japan \\ E-mail: dtk0732@mail4.doshisha.ac.jp \\ $\dagger$ Department of Intelligent Information Engineering and Science, Doshisha University \\ 1-3 Miyakodani Tatara, Kyotanabe, Kyoto 610-0321, Japan \\ E-mail: mhashimo@mail.doshisha.ac.jp
}

\begin{abstract}
This paper investigates a quantum neural network and discusses its application in control systems. A learning-type neural network-based controller that uses a multi-layer quantum neural network having qubit neurons as its information processing unit is proposed. Three learning algorithms; a back-propagation algorithm, a conjugate gradient algorithm and a real-coded genetic algorithm, are investigated to supervise the training of the multi-layer quantum neural network. To evaluate the learning performance and the capability of the quantum neural network-based controller, we conducted computational experiments for controlling a nonlinear discrete-time plant and a nonholonomic system - in this study a two-wheeled robot. The results of computational experiments confirm both the feasibility and the effectiveness of the quantum neural network-based controller and that the real-coded genetic algorithm is suitable for the learning method of the quantum neural network-based controller.
\end{abstract}

Key words : Quantum Neural Network, Qubit Neuron, Back-Propagation Algorithm, Conjugate Gradient Algorithm, Real-Coded Genetic Algorithm, Direct Controller, Nonholonomic Robot

\section{Introduction}

During the past quarter of the century, many studies about the application of both the flexibility and the learning ability of neural networks to control systems have been conducted worldwide ${ }^{(1)}$. Although many types of neural network-based control systems have been proposed $^{(2),(3)}$, several parameters of neural networks (e.g. a large number of weights between neurons) are yet to be optimized to achieve higher training performance of neural networks in practical applications.

The possibility of using quantum mechanical system for reasonable computing was introduced by Feynman ${ }^{(4)}$. Since the first quantum computing model was proposed by Deutsch ${ }^{(5)}$, quantum computing algorithms, such as Shor's factoring algorithm ${ }^{(6)}$ and Grover's search algorithm ${ }^{(7)}$, have been proposed. After the concept of quantum neural computing was originally presented by $\mathrm{Kak}^{(8)}$, interest in artificial neural networks based on quantum theoretical concepts and techniques (hereafter called quantum neural networks) increased because of the belief that quantum neural networks may provide a new understanding of certain brain functions and also help solve classically intractable problems ${ }^{(9)}$. In quantum computing, 'qubits' (an abbreviation for quantum bits) of quantum computers are the counterparts of 'bits' of classical computers and they are used to store the states of circuits in quantum computations. The two quantum states express one bit of information: $|0\rangle \in \mathbf{C}$ corresponds to the classical com-

${ }^{*}$ Received 12 Oct., 2011 (No. 11-0624) [DOI: 10.1299/jamdsm.6.526]

Copyright (c) 2012 by JSME 
puter's bit 0 and $|1\rangle \in \mathbf{C}$ corresponds to bit 1 . Here the symbol |\rangle is part of the Dirac notation. The qubit state $|\psi\rangle$ maintains a coherent superposition of states: $|\psi\rangle=a|0\rangle+b|1\rangle$, where $a$ and $b$ are complex numbers called the probability amplitudes that satisfy $|a|^{2}+|b|^{2}=1$. A multi-layer quantum neural network that utilizes qubit neurons as an information processing unit was proposed ${ }^{(10),(11)}$, where the qubit neuron model is one in which the neuron states are connected to the quantum states and the transitions between neuron states are based on operations derived from quantum logic gates. In the qubit neuron model, the neural state of firing or non-firing corresponds to the qubit state of $|1\rangle$ or $|0\rangle$, respectively, and the state of an arbitrary neuron is a coherent superposition of the two. The high learning ability of the multi-layer quantum neural network with qubit neurons was demonstrated in several basic benchmark tests $^{(12),(13)}$, such as the parity check problem, the XOR problem and the function estimation problem. It was also tested on some applications such as image processing ${ }^{(14),(15)}$, pattern recognition ${ }^{(16),(17)}$, time series prediction ${ }^{(18),(19)}$ and control of an inverted pendulum ${ }^{(20)}$. In these works, the training of the multi-layer quantum neural network is conducted using the back-propagation algorithm, which is based on the steepest descent method to minimize a quadratic cost function. However, similar to conventional neural networks, its learning occasionally falls into a local minimum depending on the search surface of the target function that is optimized in the training process. From the viewpoint of neural network-based control systems, the high learning ability of the multi-layer quantum neural network with qubit neurons would be effective to achieve controlling of a wide class of system, e.g. dynamic systems and nonlinear systems. However, studies on applying the multi-layer quantum neural network to control applications have not yet been completed. Moreover there have been few studies except for the relevant related work ${ }^{(20)}$. Although the feasibility of the multi-layer quantum neural network for controlling the inverted pendulum has been demonstrated in that work, a priori knowledge of controlling the inverted pendulum system was required in designing the controller and its training rules in order to guarantee the convergence of the multi-layer quantum neural network's training and to complete the control task. Thus the characteristics of applying the multi-layer quantum neural network to control systems have not been clarified, yet. In the control applications of neural networks, a priori knowledge of the target systems and tasks are usually not required because the neural network is expected to achieve the control from the input/output data sets by using both the nonlinear mapping and learning abilities of the neural network. By considering these characteristics of the neural network-based control systems, it is therefore important to clarify a method for designing a control system based on the multi-layer quantum neural network with qubit neurons and to evaluate its performance.

This paper investigates the capability of a multi-layer quantum neural network with qubit neurons and discusses its application in control systems. In section 2, a multi-layer quantum neural network that uses qubit neurons as its information processing unit is described. In order to improve learning performance, a conjugate gradient algorithm and a real-coded genetic algorithm are applied instead of the back-propagation algorithm for supervised training of the multi-layer quantum neural network. In section 3, a neural network-based control system using the multi-layer quantum neural network is designed. In section 4 , computational experiments for controlling a discrete time nonlinear plant using the control system based on the multi-layer quantum neural network are conducted. Computational experiments for controlling a nonholonomic system - in this case, a two-wheeled robot - are described in section 5 to evaluate the feasibility and the characteristics of this network for practical control applications.

\section{Multi-layer Quantum Neural Network with Qubit Neuron}

The operations of the rotation gate and the controlled NOT gate in quantum computations can be expressed by the quantum state rewritten using the phase $\phi$. The rotation gate, which is a phase-shift gate that transforms the phase of quantum states, can be expressed as $f\left(\phi_{1}+\phi_{2}\right)=$ $f\left(\phi_{1}\right) f\left(\phi_{2}\right)$, where $f(\phi)=\mathrm{e}^{\mathrm{i} \phi}$ (i is an imaginary unit). The controlled NOT gate, which is the 
phase reverse operation defined with respect to the controlled input parameter $\gamma$, can be given by $f(\gamma \pi / 2-\phi)$, where $\gamma=1$ and $\gamma=0$ correspond to reversal rotation and non-rotation, respectively. Although the phase of the probability amplitude of quantum state $|1\rangle$ is reversed when $\gamma=0$, this case can be treated as non-rotation since its observed probability is invariant. By considering these gates, the state of the $j$-th qubit neuron model in the $m$-th sets $z_{j}^{m}$ is defined as follows:

$$
\left\{\begin{aligned}
z_{j}^{m} & =f\left(y_{j}^{m}\right) \\
y_{j}^{m} & =\frac{\pi}{2} h\left(\delta_{j}^{m}\right)-\arg \left(v_{j}^{m}\right) \\
v_{j}^{m} & =\sum_{l} f\left(\theta_{l, j}^{m}\right) f\left(z_{l}^{m-1}\right)-f\left(\lambda_{j}^{m}\right)
\end{aligned}\right.
$$

Here, $z_{l}^{m-1}$ is the input from the $l$-th neuron in the $(m-1)$-th sets, $\delta_{j}^{m}$ is the reversal parameter corresponding to the controlled NOT gate, $\theta_{l, j}^{m}$ is the phase parameter corresponding to the phase of the rotation gate, $\lambda_{j}^{m}$ is the threshold parameter and $h()$ is a function having a range $[0,1]$ (e.g., sigmoid function $h(x)=1 /\left(1+\mathrm{e}^{-x}\right)$ ).

The multi-layer quantum neural network is designed by combining the qubit neurons in layers. In the input layer (indicated by the superscript $m$ of $I$ ), the network input $x_{l}$ in the range $[0,1]$ is first converted into the quantum states with phase in the range $[0, \pi / 2]$, and then the output given by $z_{l}^{I}=f\left(\pi x_{l} / 2\right)$ is fed into the neurons in the hidden layer (indicated by the superscript $m$ of $H$ ). In the hidden and output layers, the outputs from neurons are given by Eq. (1). By considering the probability of the state in which $|1\rangle$ is observed from the $j$-th neuron in the output layer (indicated by the superscript $m$ of $O$ ), the output from the network $u_{N N_{j}}$ is defined by the following form:

$$
u_{N N_{j}}=\left|\operatorname{Im}\left(z_{j}^{O}\right)\right|^{2}
$$

The training of the multi-layer quantum neural network is carried out by searching the optimal parameters $\theta_{l, j}^{m}, \delta_{j}^{m}$ and $\lambda_{j}^{m}$ so as to minimize the following cost function:

$$
\begin{aligned}
J(\boldsymbol{w}) & =\frac{1}{2} \sum_{p} \sum_{j}\left\{u_{d_{j}}(p)-u_{N N_{j}}(\boldsymbol{w}, p)\right\}^{2} \\
& =\frac{1}{2} \sum_{p} \sum_{j} e_{j}^{2}(\boldsymbol{w}, p)
\end{aligned}
$$

where $e_{j}(\boldsymbol{w}, p)=u_{d_{j}}(p)-u_{N N_{j}}(\boldsymbol{w}, p), u_{d_{j}}$ is the teaching signal for the $j$-th neuron of the $p$-th pattern and the vector $\boldsymbol{w}$ is composed of the parameters $\theta_{l, j}^{m}, \delta_{j}^{m}$ and $\lambda_{j}^{m}(m=I, H, L)$.

\subsection{Learning with the back-propagation algorithm}

The back-propagation algorithm can be applied to the training of the quantum neural network as follows:

$$
\boldsymbol{w}_{n+1}=\boldsymbol{w}_{n}-\kappa \frac{\partial J}{\partial \boldsymbol{w}_{n}}+\tau \Delta \boldsymbol{w}_{n-1}
$$

where $\kappa$ is the learning factor, $\tau$ is the momentum factor, and $\Delta \boldsymbol{w}_{n}$ is the weight increment at the $n$-th iteration. The gradient of the cost function is described in Appendix.

\subsection{Learning with the conjugate gradient algorithm}

The conjugate gradient method belongs to a class of second-order optimization methods collectively known as conjugate direction methods. Therefore, a quadratic form should approximate the cost function locally to apply the conjugate gradient method for the training of the multi-layer quantum neural network. The procedures of supervised training with the conjugate gradient algorithm are as follows ${ }^{(21)}$ :

\section{Initialization}

Unless prior knowledge on the weight vector $\boldsymbol{w}$ is available, choose the initial value $\boldsymbol{w}_{0}$ using 
a procedure similar to the back-propagation algorithm such as generating a random value.

\section{Computation}

1) For the initial value $\boldsymbol{w}_{0}$, use the back-propagation algorithm to compute the gradient vector $\boldsymbol{g}_{0}$.

2 ) Set the initial values of the direction vector $\boldsymbol{s}$ and the residual vector $\boldsymbol{r}: \boldsymbol{s}_{0}=\boldsymbol{r}_{0}=-\boldsymbol{g}_{0}$

3 ) At the time step $n$, use a line search to find the learning rate parameter $\eta_{n}$ that sufficiently minimizes $J(\eta)$, representing the cost function $J$ expressed as a function of $\eta$ for fixed values of $\boldsymbol{w}$ and $\boldsymbol{s}$.

4 ) Test to determine if the Euclidean norm of $\boldsymbol{r}_{n}$ has fallen below a specified value, that is, a small fraction of the initial value $\left\|\boldsymbol{w}_{0}\right\|$.

5 ) Update the weight vector: $\boldsymbol{w}_{n+1}=\boldsymbol{w}_{n}+\eta_{n} \boldsymbol{s}_{n}$

6 ) For $\boldsymbol{w}_{n+1}$, use the back-propagation algorithm to compute the update gradient vector $\boldsymbol{g}_{n+1}$.

7) Set $\boldsymbol{r}_{n+1}=-\boldsymbol{g}_{n+1}$.

8 ) Use the Poker-Ribiere method to calculate a scaling factor $\xi_{n+1}$ :

$\xi_{n+1}=\max \left\{0, \frac{\boldsymbol{r}_{n+1}^{\mathrm{T}}\left(\boldsymbol{r}_{n+1}-\boldsymbol{r}_{n}\right)}{\boldsymbol{r}_{n}^{\mathrm{T}} \boldsymbol{r}_{n}}\right\}$

9 ) Update the direction vector: $\boldsymbol{s}_{n+1}=\boldsymbol{r}_{n+1}+\xi_{n+1} \boldsymbol{s}_{n}$

10) Set time step $n=n+1$ and return to step 3 .

\section{Stopping criterion}

Terminate the algorithm when the following condition is satisfied: $\left\|\boldsymbol{r}_{n}\right\| \leq \rho\left\|\boldsymbol{r}_{0}\right\|$, where $\rho$ is a prescribed small number.

Although the line search described above is required to define the learning rate parameter $\eta$ in step 3, the learning rate parameter is estimated in the following manner ${ }^{(22)}$. Applying the Taylor expansion to the cost function $J\left(\boldsymbol{w}_{n}\right)$ around the search point $\left(\boldsymbol{w}_{n}+\eta_{n} \boldsymbol{s}_{n}\right)$ yields

$$
\begin{aligned}
J\left(\boldsymbol{w}_{n}+\eta_{n} \boldsymbol{s}_{n}\right) & =J\left(\boldsymbol{w}_{n}\right)+\eta_{n} \nabla^{\mathrm{T}} J\left(\boldsymbol{w}_{n}\right) \boldsymbol{s}_{n}+\frac{1}{2} \eta_{n}^{2} \boldsymbol{s}_{n}^{\mathrm{T}} \nabla^{2} J\left(\boldsymbol{w}_{n}\right) \boldsymbol{s}_{n}+O\left(\boldsymbol{s}_{n}^{3}\right) \\
& =J_{w}\left(\eta_{n}\right)+O\left(\boldsymbol{s}_{n}^{3}\right) .
\end{aligned}
$$

The function $J_{w}\left(\eta_{n}\right)$ can be considered as a quadratic approximation of the cost function $J\left(\boldsymbol{w}_{n}\right)$ around the search point $\left(\boldsymbol{w}_{n}+\eta_{n} \boldsymbol{s}_{n}\right)$ if the higher order terms of $O\left(\boldsymbol{s}_{n}^{3}\right)$ were negligibly small. The derivative of the function $J_{w}\left(\eta_{n}\right)$ with respect to the parameter $\eta_{n}$ is given by the following form:

$$
J_{w}^{\prime}\left(\eta_{n}\right)=\nabla^{\mathrm{T}} J\left(\boldsymbol{w}_{n}\right) \boldsymbol{s}_{n}+\eta_{n} \boldsymbol{s}_{n}^{\mathrm{T}} \nabla^{2} J\left(\boldsymbol{w}_{n}\right) \boldsymbol{s}_{n}
$$

Setting $J_{w}^{\prime}\left(\eta_{n}\right)=0$ yields

$$
\begin{aligned}
\hat{\eta}_{n} & =-\frac{\nabla^{\mathrm{T}} J\left(\boldsymbol{w}_{n}\right) \boldsymbol{s}_{n}}{\boldsymbol{s}_{n}^{\mathrm{T}} \nabla^{2} J\left(\boldsymbol{w}_{n}\right) \boldsymbol{s}_{n}} \\
& =-\frac{J_{w}^{\prime}(0)}{J_{w}^{\prime \prime}(0)}
\end{aligned}
$$

Here the values of the first and second derivatives, $J_{w}^{\prime}(0)$ and $J_{w}^{\prime \prime}(0)$, respectively, can be calculated from the quantum neural network.

$$
\begin{aligned}
& J_{w}^{\prime}(0)=-\sum_{p} \sum_{j} e_{j}\left(\boldsymbol{w}_{n}, p\right) \nabla^{\mathrm{T}} u_{N N_{j}}\left(\boldsymbol{w}_{n}, p\right) \boldsymbol{s}_{n} \\
& J_{w}^{\prime \prime}(0)=\sum_{p} \sum_{j}\left[\left\{\nabla^{\mathrm{T}} u_{N N_{j}}\left(\boldsymbol{w}_{n}, p\right) \boldsymbol{s}_{n}\right\}^{2}+e_{j}\left(\boldsymbol{w}_{n}, p\right) \boldsymbol{s}_{n}^{\mathrm{T}} \nabla^{2} u_{N N_{j}}\left(\boldsymbol{w}_{n}, p\right) \boldsymbol{s}_{n}\right]
\end{aligned}
$$

As a result, the optimal learning rate parameter $\hat{\eta}_{n}$ can be estimated.

The conjugate gradient algorithm is effective to speed up the training of the multi-layer quantum neural network; however, its training result is seriously affected by the initial values of the vector $\boldsymbol{w}_{0}$, if the locally quadratic approximation of the target function does not 
have sufficient accuracy. Since the training process with the conventional conjugate gradient algorithm continues until it reaches the conventionally determined number of times, even if the quadratic approximation of the target function does not have sufficient accuracy, the training performance would sometimes worsen. To overcome this problem, the accuracy of the quadratic approximation is evaluated by the following function $E$ in the time step $n$ to restart the training:

$$
\begin{aligned}
E_{n} & =\frac{J\left(\boldsymbol{w}_{n}\right)-J\left(\boldsymbol{w}_{n}+\hat{\eta}_{n} \boldsymbol{s}_{n}\right)}{J\left(\boldsymbol{w}_{n}\right)-J_{w}\left(\hat{\eta}_{n}\right)} \\
& =\frac{2 J_{w}^{\prime}(0)\left\{J\left(\boldsymbol{w}_{n}\right)-J\left(\boldsymbol{w}_{n}+\hat{\eta}_{n} \boldsymbol{s}_{n}\right)\right\}}{\left\{J_{w}^{\prime \prime}(0)\right\}^{2}}
\end{aligned}
$$

This function indicates the ratio of the decrease in the target function and the approximation residual. The greater the accuracy of the locally quadratic approximation of the target function, the closer the value of function $E_{n}$ is to 1 . It would be impossible to decrease the target function based on local quadratic approximation if the value tends to 0 . In step 10 , the calculation procedure will be restarted with a new initial value $\boldsymbol{w}_{0}$ if the function $E_{n}$ is smaller than the threshold value.

\subsection{Learning with the real-coded genetic algorithm}

To find the vector $\boldsymbol{w}$ that minimizes the cost function $J(\boldsymbol{w})$, the real-coded genetic algorithm $^{(23)}$ that is powerful enough to solve real parameter optimization problems of multimodality, parameter dependency, and ill-scale can also be utilized in the training of the multilayer quantum neural network. Thus, the parameter values of the vector $\boldsymbol{w}$ are directly used as gene parameters of an individual. The real-coded genetic algorithm is composed of a multiparental crossover and a generation alternation model. A real-coded ensemble crossover is used as the multi-parental crossover. The real-coded ensemble crossover is a generalization of the enhanced unimodal normal distribution crossover and has some probability distribution in the multi-parental crossover operation in order to avoid the asymmetry and bias of children distribution. In the real-coded ensemble crossover, the new individuals (children), $\boldsymbol{g}_{c}$, are generated by using multi-parental individuals, $\boldsymbol{g}_{j}(j=1,2, \cdots, N+K: N$ is the dimension of the problem, in this study it is the dimension of the vector $\boldsymbol{w}$. The value of $K$ is defined in the range of $1 \leq K \leq N_{p}-N$ where $N_{p}$ is the number of population, in this study it is found by trial and error so as to be the minimum number to make the cost function minimize.), as follows:

$$
\boldsymbol{g}_{c}=\boldsymbol{g}_{0}+\sum_{j=1}^{N+K} v_{j}\left(\boldsymbol{g}_{j}-\boldsymbol{g}_{0}\right)
$$

where $\boldsymbol{g}_{0}$ indicates the centre of gravity of the parents and $v_{j}$ is the stochastic variable that follows the probability distribution $\varphi\left(0, \sigma_{v}^{2}\right)$ where $\sigma_{v}^{2}=1 /(N+K)$. As the generation alternation model, the just generation gap which replaces parents with children completely every

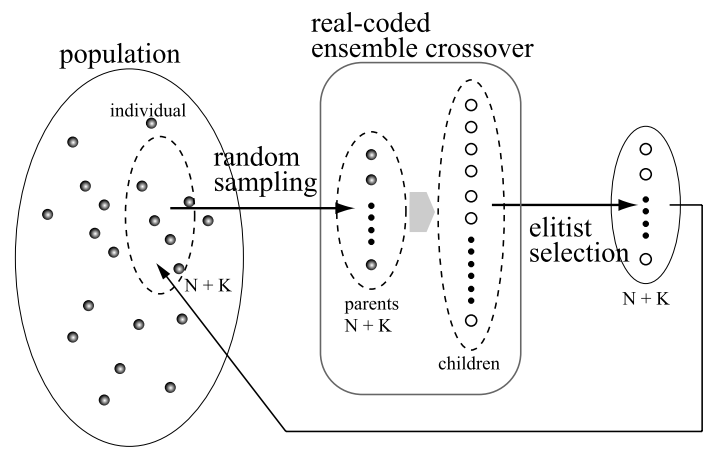

Fig. 1 Generation alternation model of the just generation gap. 
generation, as shown in Fig. 1 is utilized. In the just generation gap, the numbers of the population, parents and children are recommended to be $(15 \sim 50) N, N+K$ and $10 N$, respectively. To evaluate the individuals, a fitness function of the $q$-th individual at the $n$-th generation is defined by the reciprocal of the cost function $J\left(\boldsymbol{w}_{n}^{q}\right)$.

\section{Quantum Neural Network Controller}

To apply the multi-layer quantum neural network to controlling systems, the outputs from the multi-layer quantum neural network are converted from the range $[0,1]$ to $\left[u_{\min }, u_{\max }\right]$ with a gain and shift factors: $u_{j}=c_{0}\left(u_{N N_{j}}-u_{0}\right)$ where $u$ indicates the control input, $c_{0}$ is the gain factor and $u_{0}$ is the shift factor.

In general, neural network-based controllers have two types of training ${ }^{(24)}$ : an adaptive type in which the outputs from the neural network follow the desired outputs within one trial in a real-time process; and a learning type in which the outputs from the neural network approximate the desired outputs after several trials in an offline process. In this study, the learning-type controller is considered since either the conjugate gradient algorithm or the realcoded genetic algorithm is performed in off-line mode. Hereafter, the learning-type neural network-based controller using the multi-layer quantum neural network with qubit neurons is called the quantum neural controller.

\subsection{Direct controller}

To design the quantum neural controller, the following single-input single-output discrete-time plant is considered as a controlling target plant:

$$
y(k+d)=F_{p}\left[y(k), \cdots, y\left(k-n_{p}+1\right), u(k), \cdots, u\left(k-m_{p}\right)\right]
$$

where $y$ is the plant output, $u$ is the plant input, $n_{p}$ and $m_{p}$ are the plant orders, $k$ is the sampling number, $d$ is the dead time of the plant and $F_{p}()$ is the function that expresses the plant dynamics. The plant output $y(k)$ depends on the past plant input and output. The plant orders determine the period in which the plant output depends on them. This period is usually shorter than the trial period. This design uses the following assumptions: the upper limit orders of the plant are known and the dead time of the plant is known.

By considering the desired plant output $y_{d}(k)$, the output error $\epsilon(k)$ can be defined: $\epsilon(k)=$ $y_{d}(k)-y(k)$. When the output from the multi-layer quantum neural network is input to the plant directly, i.e. $u(k)=u_{N N}$, the multi-layer quantum neural network approximates the inverse model of the plant and such a controller is called a direct controller. Substituting Eq. (12) into the output error, an input vector $\boldsymbol{x}$ of the multi-layer quantum neural network can be defined as follows:

$$
\boldsymbol{x}(k)=\left[\begin{array}{lllrl}
y_{d}(k+d) & y(k) & \cdots & y\left(k-n_{p}+1\right) \\
& u(k-1) & \cdots & u\left(k-m_{p}\right)
\end{array}\right]^{\mathrm{T}}
$$

Figure 2 shows the block diagram of the direct quantum neural controller. However, such a direct controller makes it difficult to guarantee the stability of the control system if the training of the multi-layer quantum neural network does not converge. Although the training performance of the multi-layer quantum neural network is higher than the conventional neural networks that have a sigmoid function or a radial basis function in its neuron unit, the stability of the control system using the multi-layer quantum neural network cannot always be guaranteed at the start of the training process.

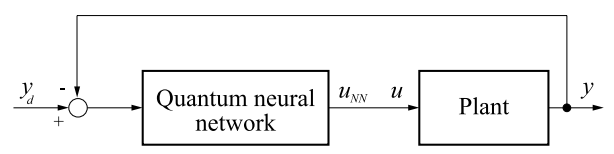

Fig. 2 Block diagram of the direct controller. 


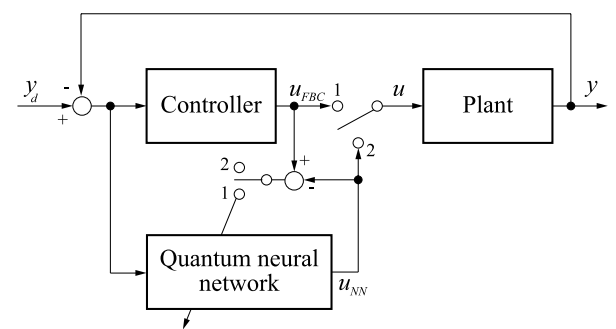

Fig. 3 Block diagram of the direct transfer function identifier-based controller.

\subsection{Direct transfer function identifier-based controller}

If a conventional feedback controller that guarantees both the stability of the control system and achievement of the control task can be found, another direct controller in which the multi-layer quantum neural network identifies the direct transfer function of the conventional feedback controller can be designed to avoid the instability problem of the direct controller. The transfer function of the feedback controller is given by:

$$
u(k+d)=F_{c}\left[u(k), \cdots, u\left(k-n_{c}+1\right), \epsilon(k), \cdots, \epsilon\left(k-m_{c}\right)\right]
$$

where $\epsilon$ is the feedback controller input, $n_{c}$ and $m_{c}$ are the controller orders and $F_{c}()$ is the function that expresses the controller dynamics. By considering Eq. (14), the input vector $x$ of the multi-layer quantum neural network can be defined as follows:

$$
\begin{array}{rrr}
\boldsymbol{x}(k)=\left[\begin{array}{rrrr}
u(k-d) & \cdots & u\left(k-d-n_{c}+1\right) \\
\epsilon(k-d) & \cdots & \epsilon\left(k-d-m_{c}\right)
\end{array}\right]^{\mathrm{T}}
\end{array}
$$

Figure 3 shows the block diagram of the direct quantum neural controller in which its training process can be divided into two steps: 1) identification (training); the control input to the plant is connected to terminal $\left.1\left(u=u_{F B C}\right), 2\right)$ control; the control input to the plant is connected to terminal $2\left(u=u_{N N}\right)$.

\section{Computational Experiments of Quantum Neural Controller}

The quantum neural controller is simulated using a discrete-time nonlinear plant to verify its feasibility. The equation of the plant in which the second-order system is dominant is as follows $^{(24)}$ :

$$
\begin{aligned}
y(k+1)=F_{s}\left[-a_{1} y(k)-a_{2} y(k-1)+b_{1} u(k)\right. & +b_{2} u(k-1) \\
& \left.+a_{3} y(k-2)+c_{n o n} y^{2}(k)\right]
\end{aligned}
$$

where $a_{3}$ and $c_{\text {non }}$ are the coefficients of the parasitic term and the nonlinear term. The values of the plant parameters were set to $a_{1}=-1.3, a_{2}=0.3, b_{1}=1, b_{2}=0.7, a_{3}=0.03$ and $c_{\text {non }}=0.2$. In this experiment, the following saturation function $F_{s}()$ is introduced in order to guarantee the boundedness of the plant output.

$$
F_{s}(x)=\left\{\begin{array}{cc}
1 & (x \geq 1) \\
x & (-1<x<1) \\
-1 & (x \leq-1)
\end{array} .\right.
$$

In this experiment, the direct controller was considered. When designing the direct controller, the plant was assumed to be linear: $d=1, n_{p}=2$ and $m_{p}=1$. Thus, the input vector of the multi-layer quantum neural network is $\boldsymbol{x}(k)=\left[\begin{array}{lllll}y_{d}(k+1) & y(k) & y(k-1) & u(k-1)\end{array}\right]^{\mathrm{T}}$. The number of qubit neurons in the hidden layer was set to 4 and the gain factor $c_{0}$ and the shift factor $u_{0}$ were 2 and 0.5 , respectively. The training conditions of the quantum neural controller were as follows: 1) the parameters in the back-propagation algorithm were set to $\kappa=10^{-3}$ and $\tau=0.9$ and the training iteration of the quantum neural network for the training patterns was limited to $10^{4}$ with the maximum allowable cost function of $0.1,2$ ) the parameter 
in the stopping criterion of the conjugate gradient algorithm was set to $\rho=10^{-5}$ and the maximum allowable cost function is 0.1 , while the number of iterations for the training patterns was limited to $10^{4}$ and 3 ) in the real-coded genetic algorithm, the dimension of the problem $N$ was $30, K$ was 1 , the total number of generation was 2000 with the maximum allowable cost function of 0.1 and the probability distribution $\varphi$ was the uniform distribution $\mathcal{U}$ in the range $[-1,1]$. In the experiments, a success of the quantum neural controller's learning is defined when the cost function becomes lower than the maximum allowable cost function. In the training process of either the back-propagation algorithm or the conjugate gradient algorithm, the information of a system Jacobian $\partial y / \partial u$ is required in order to calculate the derivative of the cost function. To simplify the experiments, the system Jacobian was assumed to be 1 by using a priori knowledge of the plant. In the training process and the open test of the quantum neural controller, the desired plant output $y_{d}(k)$ was a rectangular wave so as to take account of the frequency richness. The number of samples within one cycle of the rectangular wave was 50 and the amplitude of the wave was changed randomly in the range $[0,1]$.

Figures 4,5 and 6 are examples of the open testing responses trained by the backpropagation algorithm, the conjugate gradient algorithm and the real-coded genetic algorithm, respectively. In each figure, the upper part shows the desired plant output $y_{d}(k)$ and the plant output $y(k)$; and the lower part indicates the control input $u(k)$ (output from the multi-layer quantum neural network). As shown in each figure, the plant output tracks the desired output. The quantum neural controller can achieve the control task to control the nonlinear plant even though a small tracking error remains. These results indicate the feasibility of the quantum neural controller. However, the success rate of the quantum neural controller's learning are low if the back-propagation algorithm is utilized since the training process greatly depends on the initial value $\boldsymbol{w}_{0}$. The brute-force approach was required to find the initial value $\boldsymbol{w}_{0}$ that makes the quantum neural controller's learning proceed. In the conjugate gradient algorithm, the restarted calculation procedure with a new initial value $\boldsymbol{w}_{0}$ is also required many times. By comparing the three algorithms, the real-coded genetic algorithm suits the learning of the quantum neural controller since it does not require the system Jacobian information in the training process and the success rate of learning by the real-coded genetic algorithm is high. Furthermore, the control performance of the quantum neural controller trained by the real-coded genetic algorithm is better than those achieved by other algorithms.

\section{Computational Experiments for Controlling a Nonholonomic System with a Quantum Neural Network Controller}

To investigate the characteristics of the quantum neural controller, computational simulations of controlling a nonholonomic system - in this experiment, a position/orientation control of a two-wheeled robot - were conducted. In this experiment, a direct transfer function identifier-based controller was considered as the quantum neural network controller.

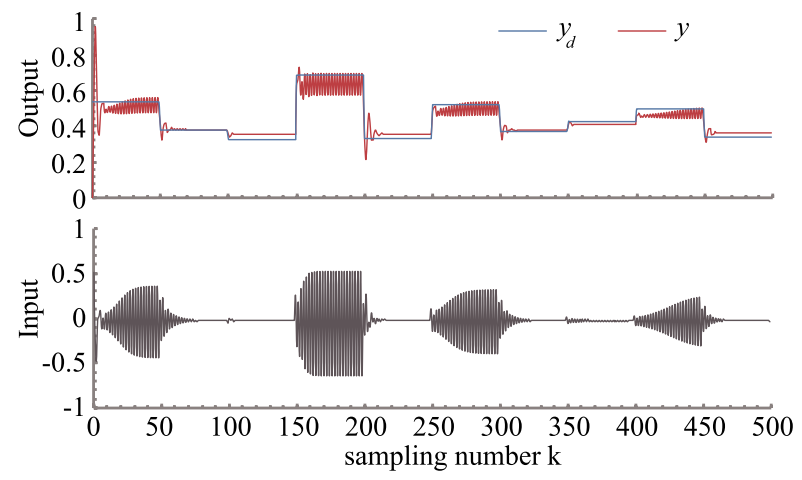

Fig. 4 Open testing response of system trained by the back-propagation algorithm where the cost function of the open test is $J=0.657$ (top: desired and system outputs, bottom: control input). 


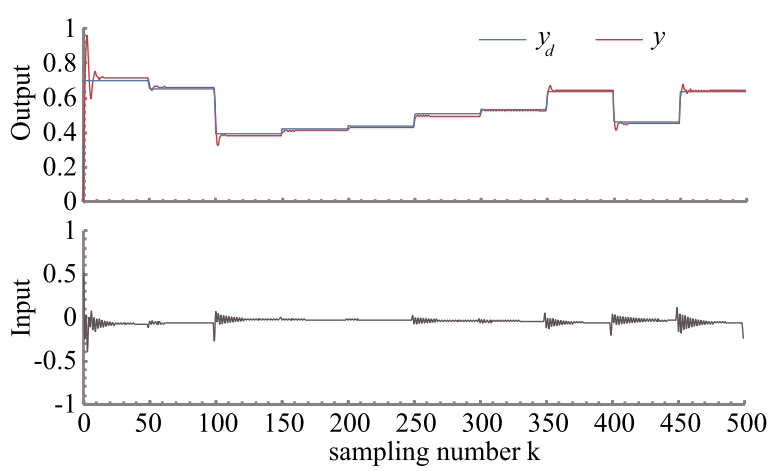

Fig. 5 Open testing response of system trained by the conjugate gradient algorithm where the cost function of the open test is $J=0.362$ (top: desired and system outputs, bottom: control input).

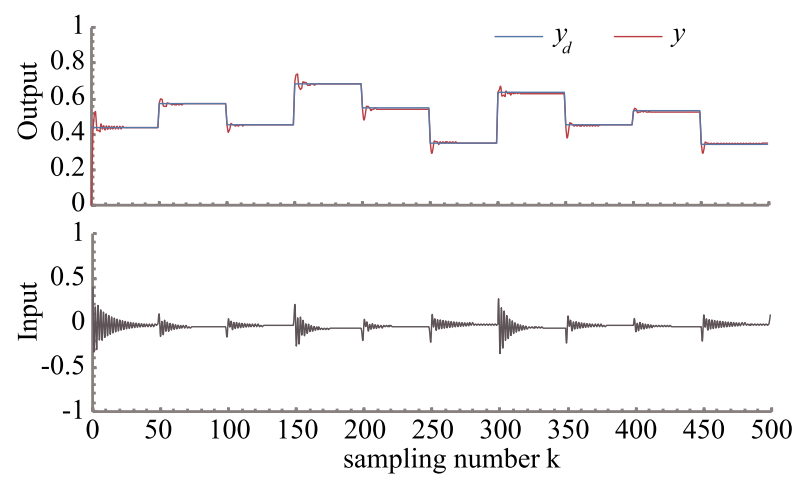

Fig. 6 Open testing response of system trained by the real-coded genetic algorithm where the cost function of the open test is $J=0.127$ (top: desired and system outputs, bottom: control input).

The two-wheeled robot shown in Fig. 7 was used as a plant controlled by the quantum neural controller. Here, the position and orientation of the two-wheeled robot at time $t$ is indicated by $\left[\begin{array}{ll}x_{R}(t) & y_{R}(t)\end{array}\right]$ and $\zeta_{R}(t)$, respectively. The kinematic equation of the twowheeled robot is given as follows:

$$
\left[\begin{array}{c}
\dot{x}_{R}(t) \\
\dot{y}_{R}(t) \\
\dot{\zeta}_{R}(t)
\end{array}\right]=\left[\begin{array}{cc}
\cos \zeta_{R}(t) & 0 \\
\sin \zeta_{R}(t) & 0 \\
0 & 1
\end{array}\right]\left[\begin{array}{c}
v_{p}(t) \\
v_{r}(t)
\end{array}\right]
$$

where $v_{p}(t)$ and $v_{r}(t)$ are the translational and rotational angular velocities of the two-wheeled robot, respectively. By introducing an input transform and a state vector transform into Eq. (17), the modified error system ${ }^{(25)}$ can be obtained as follows:

$$
\left[\begin{array}{c}
\dot{z}_{c_{1}}(t) \\
\dot{z}_{c_{2}}(t) \\
\dot{z}_{c_{3}}(t)
\end{array}\right]=\left[\begin{array}{c}
\mu_{1}(t) \\
\mu_{2}(t) \\
\mu_{1}(t) z_{c_{2}}(t)
\end{array}\right]
$$

where

$$
\begin{aligned}
& \boldsymbol{z}_{c}(t)=\boldsymbol{x}_{c}(t)-\boldsymbol{x}_{d}, \\
& \boldsymbol{x}_{c}(t)=\left[\begin{array}{lll}
\zeta_{R}(t) & -x_{R}(t) \cos \zeta_{R}(t)-y_{R}(t) \sin \zeta_{R}(t) & -x_{R}(t) \sin \zeta_{R}(t)+y_{R}(t) \cos \zeta_{R}(t)
\end{array}\right]^{\mathrm{T}}, \\
& \boldsymbol{x}_{d}=\left[\begin{array}{lll}
\zeta_{e} & -x_{e} \cos \zeta_{e}-y_{e} \sin \zeta_{e} & -x_{e} \sin \zeta_{e}+y_{e} \cos \zeta_{e}
\end{array}\right. \\
& \left.+\left(-x_{e} \cos \zeta_{e}-y_{e} \sin \zeta_{e}\right)\left\{\zeta_{R}(t)-\zeta_{e}\right\}\right]^{\mathrm{T}}, \\
& \mu_{1}(t)=v_{r}(t) \text { and } \\
& \mu_{2}(t)=-v_{p}(t)-\left\{-x_{R}(t) \sin \zeta_{R}(t)+y_{R}(t) \cos \zeta_{R}(t)\right\} .
\end{aligned}
$$

Here, $\left[\begin{array}{ll}x_{e} & y_{e}\end{array}\right]$ and $\zeta_{e}$ are the final position and orientation of the two-wheeled robot, respectively. 


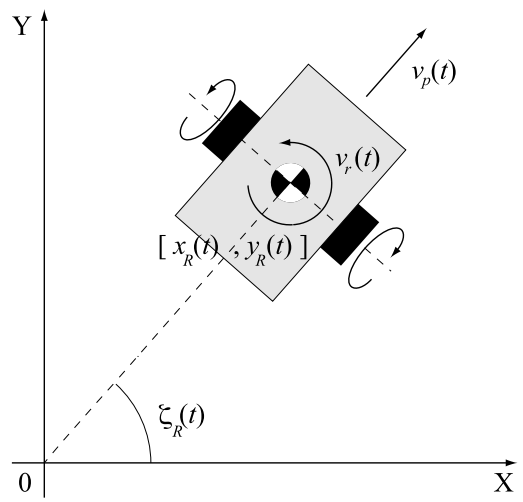

Fig. 7 Configuration of two-wheeled robot.

The discontinuous state feedback control law, a method to exponentially stabilize nonholonomic chained systems with two control inputs proposed by Astolfi( ${ }^{(26)}$ is utilized as the conventional feedback controller that the quantum neural network identifies. The state feedback control law to the chained system Eq. (18) is given by the following forms when $z_{c_{1}}(t) \neq 0$ :

$$
\left\{\begin{array}{l}
\mu_{1}(t)=-\alpha z_{c_{1}}(t) \\
\mu_{2}(t)=\beta_{2} z_{c_{2}}(t)+\beta_{3} \frac{z_{c_{3}}(t)}{z_{c_{1}}(t)}
\end{array}\right.
$$

where $\alpha>0$ and $\beta_{i}$ satisfies $\sigma\left(\left[\begin{array}{cc}\beta_{2} & \beta_{3} \\ -\alpha & \alpha\end{array}\right]\right) \subset \mathbf{C}^{-}$. Here $\sigma(\boldsymbol{A})$ denotes the spectrum of the matrix $\boldsymbol{A}$ and $\mathbf{C}^{-}$denotes the open left-half complex plane.

In the computational experiments, the parameters of the state feedback control law were as follows: $\alpha=1, \beta_{2}=-5$ and $\beta_{3}=9$. The plant composed of the model of the twowheeled robot and the state feedback control law was digitized with a sampling rate of 10 ms. The training patterns of the quantum neural controller were generated by the numerical simulation in which the wheeled-robot is controlled within 200 sampling numbers from 16 initial conditions: $\left[\begin{array}{lll}x_{R}(0) & y_{R}(0) & \zeta_{R}(0)\end{array}\right]=\left[\begin{array}{llll} \pm 1 & \pm 1 & m \pi / 4\end{array}\right]$ and $\left[\begin{array}{lll} \pm 1 & \mp 1 & m \pi / 4\end{array}\right]$ where $m= \pm 1$ and \pm 3 to the origin $\left(x_{e}=0, y_{e}=0\right.$ and $\left.\zeta_{e}=0\right)$ using the discrete plant. Since the training patterns were resampled with a sampling interval of $100 \mathrm{~ms}$, the total number of training patterns is 320 . In the multi-layer quantum neural network, the number of qubit neurons in the input layer was three and that in the output layer was two; this is because the multi-layer quantum neural network approximates the state feedback control law of Eq. (19). Thus, the input vector of the multi-layer quantum neural network is given by $\boldsymbol{x}(k)=$

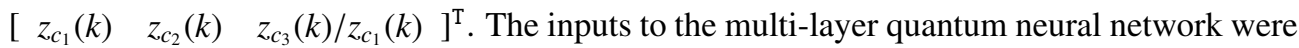
scaled by a scale factor and adjusted in the range $[0,1]$. In the experiments, the scale factor was $2 \pi$ and the gain factor $c_{0}$ and the shift factor $u_{0}$ were 50 and 0.5 , respectively. These values were determined by trial and error to achieve the convergence of the multi-layer quantum neural network's training. The training conditions of the quantum neural controller were as follows: 1) the parameters in the back-propagation algorithm were set to $\kappa=10^{-3}$ and $\tau=0.9$ and the training iteration of the quantum neural network for the training patterns was limited to $10^{4}$ with the maximum allowable cost function of $\left.10^{-3}, 2\right)$ the parameter in the stopping criterion of the conjugate gradient algorithm was set to $\rho=10^{-5}$ and the maximum allowable cost function was $10^{-3}$, while the number of iterations for the training patterns was limited to $10^{4}$ and 3 ) in the real-coded genetic algorithm, $K$ was 1 , the total number of generation was $10^{4}$ with the maximum allowable cost function of $10^{-3}$ and the probability distribution $\varphi$ was the uniform distribution $\mathcal{U}$ in the range $[-1,1]$.

The effect of the number of qubit neurons in the hidden layer to the cost function was simulated numerically to investigate the mapping ability of the multi-layer quantum neural 
Table 1 Relationship between number of qubit neurons in the hidden layer and the cost function.

\begin{tabular}{|c|c|c|c|}
\hline \multirow{2}{*}{$\begin{array}{c}\text { Number of } \\
\text { neurons }\end{array}$} & \multicolumn{3}{|c|}{ Mean of cost function (Standard deviation) } \\
\cline { 2 - 4 } & back-propagation algorithm & conjugate gradient algorithm & real-coded genetic algorithm \\
\hline 3 & $0.4468(0.0951)$ & $0.0543(0.0261)$ & $0.0524(0.0209)$ \\
\hline 5 & $0.1184(0.0201)$ & $0.0075(0.0016)$ & $0.0128(0.0060)$ \\
\hline 10 & $0.0648(0.0199)$ & $0.0040(0.0019)$ & $0.0010(0)$ \\
\hline 15 & $0.0561(0.0180)$ & $0.0017(0.0007)$ & $0.0010(0)$ \\
\hline
\end{tabular}

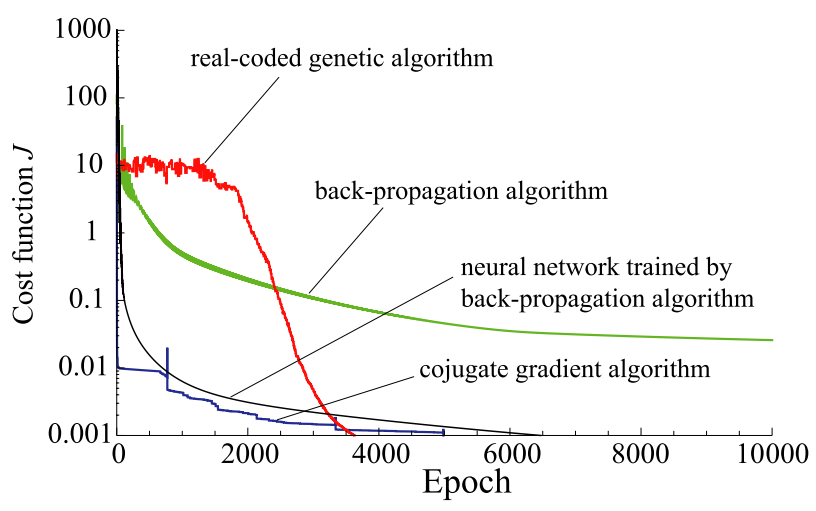

Fig. 8 Examples of multi-layer quantum neural network's training process.

network. Table 1 shows the relationship between the number of qubit neurons in the hidden layer and the cost function. The mean and standard deviation of the cost function was calculated using 10 training results for each number of the qubit neuron. If the number of qubit neurons was small (e.g. three neurons in the hidden layer), the training of the multi-layer quantum neural network often quitted at the limit number of iterations. As shown in Table 1 , the mean and standard deviation of the cost function decrease as the number of qubit neurons increase. To accurately identify the direct transfer function of the state feedback control law with the multi-layer quantum neural network, an adequate number of qubit neurons are required in the hidden layer as well as in conventional neural networks.

Figure 8 shows an example of the multi-layer quantum neural network's training process in the direct transfer function identification. Here the horizontal axis represents the epoch and the vertical axis represents the cost function. In this experiment, the number of qubit neurons in the hidden layer was set to 15 . To compare the learning ability of the multi-layer quantum neural network, the conventional neural network trained by the back-propagation algorithm was tested. The topology of the neural network was a 3-18-2 network and the sigmoid function was used in the neurons of the hidden and output layers and the linear function was utilized in the neurons of the input layer. The total number of neural network's parameters (weights and thresholds) was 109 so as to equal the number of parameters in the multi-layer quantum neural network. The parameters in the back-propagation algorithm were set to $\kappa=10^{-3}$ and $\tau=0.9$ and the training iteration of the neural network for the training patterns was limited to $10^{4}$ with the maximum allowable cost function of $10^{-3}$. The mean of the cost function obtained by the neural network is 0.0014 with standard deviation of 0.0004 for 10 training results, however, the success rate of the neural network's learning is lower than those of the multi-layer quantum neural networks. As shown in Fig. 8, the multi-layer quantum neural network trained by the real-coded genetic algorithm decreases the cost function faster and makes it smaller, as opposed to the neural network.

To investigate the control performance of the quantum neural controller, the responses to the untrained initial conditions of the two-wheeled robot were simulated after the training of the quantum neural network was converged. In this experiment, the number of qubit neurons in the hidden layer was set to 15 . Figures 9 and 10 illustrate the examples of the moving trajectories of the two-wheeled robot controlled by using the quantum neural controller trained by the conjugate gradient algorithm and the genetic algorithm, respectively. Here, each coloured tra- 


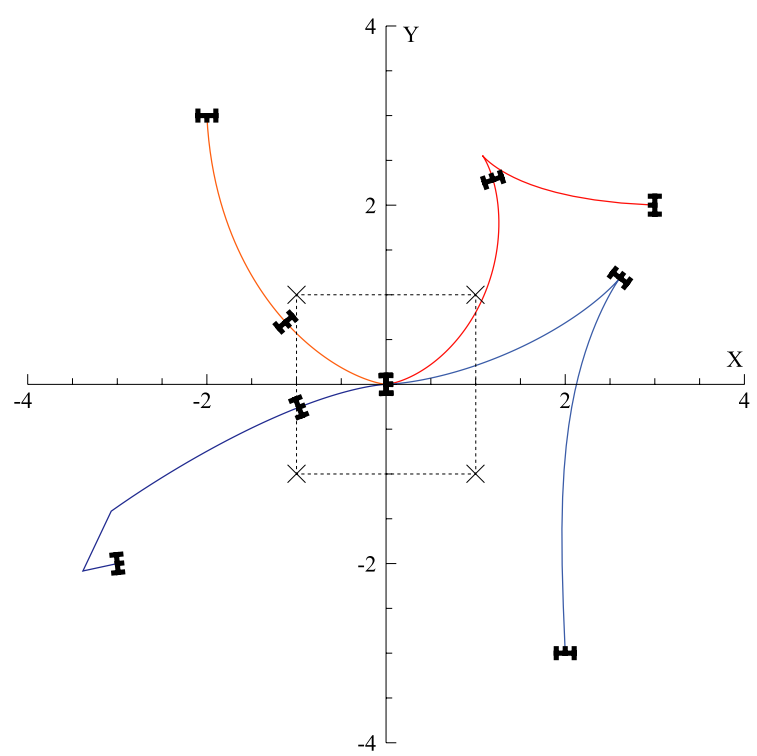

Fig. 9 Examples of the moving trajectory of the two-wheeled robot controlled by quantum neural controller that is trained by the conjugate gradient algorithm where initial positions and orientations of the two-wheeled robot are not included in the training patterns (' $x$ ' indicates the training pattern: position and orientation).

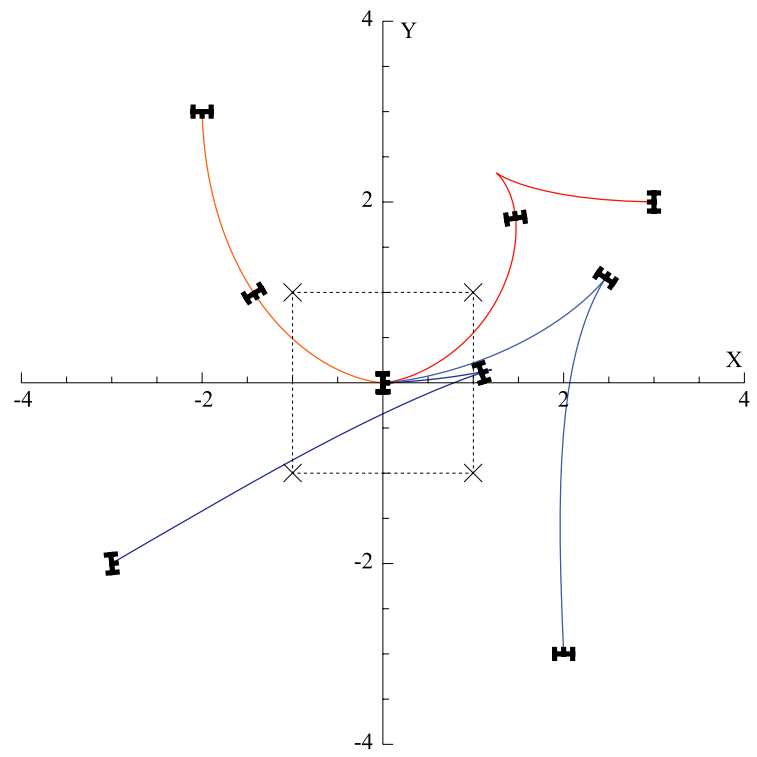

Fig. 10 Examples of the moving trajectory of the two-wheeled robot controlled by quantum neural controller that is trained by the real-coded genetic algorithm where initial positions and orientations of the two-wheeled robot are not included in the training patterns (' $x$ ' indicates the training pattern: position and orientation).

jectory corresponds to the response to each initial condition. The postures of the two-wheeled robot at the initial, transient and final positions are illustrated with the symbol 'E'. The initial conditions of the two-wheeled robot were set to [ $\left.\begin{array}{llll}x_{R}(0) & y_{R}(0) & \zeta_{R}(0)\end{array}\right]=\left[\begin{array}{lll}3 & 2 & \pi\end{array}\right]$, $\left[\begin{array}{lll}-2 & 3 & -\pi / 2\end{array}\right],\left[\begin{array}{lll}-3 & -2 & 0.1\end{array}\right]$, and [ $\left.\begin{array}{lll}2 & -3 & \pi / 2\end{array}\right]$. These figures indicate that the quantum neural controller can achieve the control task of regulating both position and orientation of the two-wheeled robot from untrained initial conditions. As shown in these figures, the two-wheeled robot can be stabilized from each initial condition into the reference - in this experiment, the origin - by using the quantum neural controllers. These results demonstrate the robustness of the quantum neural network controller and indicate that the multi-layer quantum 
neural network can identify the transfer function of the feedback controller even though the limited training pattern was used. Although similar control performances can be achieved by the quantum neural controllers, the trained vector $\boldsymbol{w}$ of the multi-layer quantum neural network is different between the conjugate gradient algorithm and the genetic algorithm. This is because the transfer function identification by the multi-layer quantum neural network is different from the parameter estimation of the target plant since the multi-layer quantum neural network achieves the approximation of the relationship between the target plant's inputs and outputs.

\section{Conclusions}

This paper investigated the capabilities of a quantum neural network and explored its application in control systems. A multi-layer quantum neural network that uses qubit neurons as an information processing unit was used to design a learning-type quantum neural network-based controller. Three learning algorithms: the back-propagation algorithm, the conjugate gradient algorithm and the real-coded genetic algorithm, were investigated as supervised training of the multi-layer quantum neural network. Computational experiments to control the nonlinear discrete-time plant and the two-wheeled robot are conducted in order to evaluate the learning performance and the capability of the quantum neural controller. The results of the computational experiments confirmed both the feasibility and effectiveness of the quantum neural controller, and the real-coded genetic algorithm was suitable for the learning method of the quantum neural controller.

\section{References}

( 1 ) K. S. Narendra and K. Parthictsarathy, "Identification and Control of Dynamics System Using Neural Networks", IEEE Transactions on Neural Networks Vol. 1, No. 1, (1990), pp. 4-27.

( 2 ) M. T. Hagan, H. B. Demuth and O. De Jesus, "An Introduction to the Use of Neural Networks in Control Systems", International Journal of Robust and Nonlinear Control, Vol. 12, No. 11, (2002), pp. 959-985.

( 3 ) J. Balderud and L. Giovanini, "Adaptive Control and Signal Processing Literature Survey”, International Journal of Adaptive Control and Signal Processing, Vol. 22, No. 3, (2008), pp. 318-321.

( 4 ) R. Feynman, "Simulating Physics with Computers", International Journal of Theoretical Physics, Vol. 21, (1982), pp. 467-488.

( 5 ) D. Deutsch, "Quantum Computational Networks", in Proceedings of the Royal Society of London, Series A, Vol. 425, (1989), pp. 73-90.

( 6 ) P. W. Shor, "Algorithms for Quantum Computation: Discrete log and factoring”. in Proceedings of the 35th Annual Symposium on the Foundations of Computer Science, (1994), pp. 124-134.

( 7 ) L. K. Grover, "A Fast Quantum Mechanical Algorithm for Database Search”, in Proceedings of the 28th Annual ACM Symposium on the Theory of Computing, (1996), pp. 212-219.

( 8 ) S. C. Kak, "On Quantum Neural Computing”, Information Science, Vol. 83, (1995), pp. 143-163.

( 9 ) A. A. Ezhov and D. Ventura, "Quantum Neural Networks", Future Directions for Intelligent Systems and Information Sciences, Springer-Verlag Heidelberg, (2000), pp. 213-234.

(10) N. Matsui, M. Takai and H. Nishimura, "A Network Model Based on Qubit-like Neuron Corresponding to Quantum Circuit", Electronics and Communications in Japan, Vol. 83, No. 10, (2000), pp. 67-73.

(11) J. L. Mitrpanont and A. Srisuphab, "The Realization of Quantum Complex-Valued Backpropagation Neural Network in Pattern Recognition Problem", in Proceedings of 
the 9th International Conference on Neural Information Processing, Vol. 1, (2002), pp. 462-466.

(12) N. Kouda, N. Matsui, H. Nishimura and F. Peper, "Qubit Neural Network and Its Learning Efficiency”, Neural Computing and Application, Vol. 14, No.2, (2005), pp. 114-121.

(13) M. Maeda, M. Suenaga and H. Miyajima, "Qubit Neural Network by Quantum Circuit for XOR Problem”, in Proceedings of International Conference on Intelligent Computing, (2005), pp. 1782-1791.

(14) K. Mori, T. Isokawa, N. Kouda, N. Matsui and H. Nishimura, "Qubit Inspired Neural Network towards Its Practical Applications", in Proceedings of 2006 International Joint Conference on Neural Networks, (2006), pp. 224-229.

(15) L. Huifang and L. Mo, "A New Method of Image Compression Based on Quantum Neural Network", in Proceedings of 2010 International Conference of Information Science and Management Engineering, (2010), pp. 567-570.

(16) F. Li, S. Zhao and B. Zheng, "Quantum Neural Network in Speech Recognition”, in Proceedings of 6th International Conference on Signal Processing, Vol. 2, (2002), pp. $1267-1270$.

(17) S. Yu and N. Ma, "Quantum Neural Network and Its Application in Vehicle Classification", in Proceedings of Fourth International Conference on Natural Computation, (2008), pp. 499-503.

(18) C. R. B. Azevedo and T. A. E. Ferreira, "Time Series Forecasting with Qubit Neural Networks", in Proceedings of the Eleventh IASTED International Conference on Artificial Intelligence and Soft Computing, (2007), pp.13-18.

(19) R. A. Araujo, A. L. I. Oliveira and S. C. B. Soares, "A Quantum-Inspired Hybrid Methodology for Financial Time Series Prediction" in Proceedings of the 2010 International Joint Conference on Neural Networks, (2010), pp. 1-8.

(20) N. Kouda, N. Matsui, H. Nishimura and F. Peper, "An Examination of Qubit Neural Network in Controlling an Inverted Pendulum", Neural Processing Letters, Vol. 22, No.3, (2005), pp. 277-290.

(21) S. Haykin, Neural Networks - A Comprehensive Foundation -, Prentice Hall, (1999).

(22) T. Yoshida, M. Gotoh and N. Tawara, "A Study on a Method Improving Efficiency of Search Based on Conjugate Gradient Method", Journal of Japan Industrial Management Association, Vol. 48, No. 5, (1997), pp. 257-263.

(23) Y. Akimoto, J. Sakuma, I. Ono and S. Kobayashi, "Adaptation of Expansion Rate for Real-coded Crossovers", in Proceedings of 11th Annual Conference on Genetic and Evolutionary Computation, (2009), pp. 739-746.

(24) T. Yamada and T. Yabuta, "Dynamic System Identification Using Neural Networks", IEEE Transactions of Systems, Man, and Cybernetics, Vol. 23, No. 1, (1993), pp. 204211.

(25) O. J. Sordalen and O. Egeland, "Exponential Stabilization of Nonholonomic Chained Systems", IEEE Transaction on Automatic Control, Vol. 40, No. 1, (1995), pp. 35-49.

(26) A. Astolfi, "Exponential Stabilization of a Car-like Vehicle", in Proceedings of IEEE International Conference on Robotics and Automation, (1995), pp. 1391-1396.

\section{Appendix: Gradient of cost function}

When the topology of the multi-layer quantum neural network has three layers, the partial derivatives of the cost function with respect to the quantum neural network's parameter in the output layer are given as follows:

$$
\begin{aligned}
\frac{\partial J}{\partial \delta_{j}^{O}} & =-\pi \sum_{p} \sum_{j} e_{j}\left|\sin y_{j}^{O}\right| \operatorname{sgn}\left(\sin y_{j}^{O}\right) \cos y_{j}^{O} h^{\prime}\left(\delta_{j}^{O}\right), \\
\frac{\partial J}{\partial \theta_{l, j}^{O}} & =2 \sum_{p} \sum_{j} e_{j}\left|\sin y_{j}^{O}\right| \operatorname{sgn}\left(\sin y_{j}^{O}\right) \cos y_{j}^{O} \frac{a_{j}^{O} \cos \left(\theta_{l, j}^{O}+y_{l}^{H}\right)+b_{j}^{O} \sin \left(\theta_{l, j}^{O}+y_{l}^{H}\right)}{\left(a_{j}^{O}\right)^{2}+\left(b_{j}^{O}\right)^{2}},
\end{aligned}
$$




$$
\frac{\partial J}{\partial \lambda_{j}^{O}}=-2 \sum_{p} \sum_{j} e_{j}\left|\sin y_{j}^{O}\right| \operatorname{sgn}\left(\sin y_{j}^{O}\right) \cos y_{j}^{O} \frac{a_{j}^{O} \cos \lambda_{j}^{O}+b_{j}^{O} \sin \lambda_{j}^{O}}{\left(a_{j}^{O}\right)^{2}+\left(b_{j}^{O}\right)^{2}}
$$

where $\operatorname{sgn}()$ is the sign function,

$$
\begin{aligned}
& a_{j}^{O}=\sum_{l} \cos \left(\theta_{l, j}^{O}+y_{l}^{H}\right)-\cos \lambda_{j}^{O} \text { and } \\
& b_{j}^{O}=\sum_{l} \sin \left(\theta_{l, j}^{O}+y_{l}^{H}\right)-\sin \lambda_{j}^{O} .
\end{aligned}
$$

The partial derivatives of the cost function with respect to the quantum neural network's parameter in the hidden layer are given as follows:

$$
\begin{aligned}
\frac{\partial J}{\partial \delta_{l}^{H}} & =\sum_{m} \frac{\partial J}{\partial \theta_{l, m}^{O}} \frac{\pi}{2} h^{\prime}\left(\delta_{l}^{H}\right), \\
\frac{\partial J}{\partial \theta_{i, l}^{H}} & =-\sum_{m} \frac{\partial J}{\partial \theta_{l, m}^{O}} \frac{a_{l}^{H} \cos \left(\theta_{i, l}^{H}+\frac{\pi}{2} x_{i}\right)+b_{l}^{H} \sin \left(\theta_{i, l}^{H}+\frac{\pi}{2} x_{i}\right)}{\left(a_{l}^{H}\right)^{2}+\left(b_{l}^{H}\right)^{2}}, \\
\frac{\partial J}{\partial \lambda_{l}^{H}} & =\sum_{m} \frac{\partial J}{\partial \theta_{l, m}^{O}} \frac{a_{l}^{H} \cos \lambda_{l}^{H}+b_{l}^{H} \sin \lambda_{l}^{H}}{\left(a_{l}^{H}\right)^{2}+\left(b_{l}^{H}\right)^{2}}
\end{aligned}
$$

where

$$
\begin{aligned}
a_{l}^{H} & =\sum_{i} \cos \left(\theta_{i, l}^{H}+\frac{\pi}{2} x_{i}\right)-\cos \lambda_{l}^{H} \text { and } \\
b_{l}^{H} & =\sum_{i} \sin \left(\theta_{i, l}^{H}+\frac{\pi}{2} x_{i}\right)-\sin \lambda_{l}^{H} .
\end{aligned}
$$

